\title{
Playing to Retain the Advantage
}

\section{Journal Article}

\section{Author(s):}

Alon, Noga; Hefetz, Dan; Krivelevich, Michael

Publication date:

2010-07

\section{Permanent link:}

https://doi.org/10.3929/ethz-b-000020677

\section{Rights / license:}

In Copyright - Non-Commercial Use Permitted

Originally published in:

Combinatorics, Probability \& Computing 19(4), https://doi.org/10.1017/S0963548310000064 


\title{
Playing to Retain the Advantage
}

\section{NOGA ALON ${ }^{1 \dagger}{ }^{\dagger}$ DAN HEFETZ ${ }^{2}$ and MICHAEL KRIVELEVICH ${ }^{3 \ddagger}$}

${ }^{1}$ Schools of Mathematics and Computer Science, Raymond and Beverly Sackler Faculty of Exact Sciences, Tel Aviv University, Tel Aviv, 69978, Israel (e-mail: nogaa@tau.ac.il)

${ }^{2}$ Institute of Theoretical Computer Science, ETH Zurich, CH-8092 Switzerland (e-mail: dan.hefetz@inf.ethz.ch)

${ }^{3}$ School of Mathematical Sciences, Raymond and Beverly Sackler Faculty of Exact Sciences, Tel Aviv University, Tel Aviv, 69978, Israel (e-mail: krivelevapost.tau.ac.il)

Received 6 February 2009; revised 3 February 2010; first published online 19 March 2010

\begin{abstract}
Let $P$ be a monotone increasing graph property, let $G=(V, E)$ be a graph, and let $q$ be a positive integer. In this paper, we study the $(1: q)$ Maker-Breaker game, played on the edges of $G$, in which Maker's goal is to build a graph that satisfies the property $P$. It is clear that in order for Maker to have a chance of winning, $G$ itself must satisfy $P$. We prove that if $G$ satisfies $P$ in some strong sense, that is, if one has to delete sufficiently many edges from $G$ in order to obtain a graph that does not satisfy $P$, then Maker has a winning strategy for this game. We also consider a different notion of satisfying some property in a strong sense, which is motivated by a problem of Duffus, Łuczak and Rödl [6].
\end{abstract}

\section{Introduction}

Let $X$ be a finite set and let $\mathcal{F} \subseteq 2^{X}$ be a family of subsets. In the $(p: q)$ Maker-Breaker game $(X, \mathcal{F})$, two players, called Maker and Breaker, take turns in claiming previously unclaimed elements of $X$, with Maker going first. The set $X$ is called the 'board' of the game and the members of $\mathcal{F}$ are referred to as the 'winning sets'. Maker claims $p$ board elements per turn, whereas Breaker claims $q$. The game ends when every board element has been claimed by some player. Maker wins the game if he occupies all elements of some winning set; otherwise Breaker wins. We say that a $(p: q)$ game $(X, \mathcal{F})$ is a Maker's win if Maker has a strategy that ensures his

$\dagger$ Research supported in part by a USA-Israel BSF grant, by a grant from the Israel Science Foundation, by an ERC Advanced Grant and by the Hermann Minkowski Minerva Center for Geometry at Tel Aviv University.

$\ddagger$ Research supported in part by USA-Israel BSF grant 2006322, by grant 1063/08 from the Israel Science Foundation, and by a Pazy memorial award. 
win in this game against any strategy of Breaker, otherwise the game is a Breaker's win. Note that $p, q, X$, and $\mathcal{F}$ determine whether the game is a Maker's win or a Breaker's win.

In this paper we are interested in the following family of Maker-Breaker games. Let $P$ be a monotone increasing graph property, let $G=(V, E)$ be a graph, and let $q$ be a positive integer. In the $(1: q)$ Maker-Breaker game $\left(E, \mathcal{F}_{P}\right)$, Maker's goal is to build a subgraph of $G$ that satisfies the property $P$, that is, $\mathcal{F}_{P}=\left\{E^{\prime} \subseteq E: G\left[E^{\prime}\right] \in P\right\}$. We are interested in characteristics of the graph $G$ which guarantee that the $(1: q)$ game $\left(E, \mathcal{F}_{P}\right)$ is a Maker's win. Since $P$ is a monotone increasing property, it is clear that Maker will lose the game $\left(E, \mathcal{F}_{P}\right)$ if $G \notin P$. On the other hand, it seems plausible that if $G$ satisfies $P$ in a 'sufficiently robust' manner, then Maker should be able to retain some of this initial advantage and win the game. This notion of 'robustness' is made precise in the following definition.

Definition. Let $\varepsilon>0$, let $P$ be a monotone increasing graph property, and let $G=(V, E)$ be a graph with $m$ edges. $G$ is said to be $\varepsilon$-robust with respect to $P$ if one has to delete at least $\mathrm{\varepsilon m}$ edges from $G$ in order to obtain a graph that does not satisfy $P$.

Remark. A graph $G$ with $m$ edges is $\varepsilon$-robust with respect to a monotone increasing property $P$ if and only if $G$ is $\varepsilon$-far from satisfying the (monotone decreasing) complement property $\bar{P}$ (that is, one has to delete at least $\varepsilon m$ edges from $G$ in order to obtain a graph that does satisfy $\bar{P}$ ).

We prove the following general result.

Theorem 1.1. Let $\varepsilon>0$, let $P$ be a monotone increasing graph property, and let $G=(V, E)$ be a graph with $n$ vertices and $m=\Theta\left(n^{2}\right)$ edges. If $G$ is $\varepsilon$-robust with respect to $P$ and $n$ is sufficiently large, then there exist positive constants $c$ and $\alpha=\alpha(\varepsilon, P)$, such that Maker has a winning strategy for the $(1: q)$ game $\left(E, \mathcal{F}_{P}\right)$ for every $q \leqslant c n^{\alpha}$.

The bound on $q$ given in Theorem 1.1 is clearly best possible up to the value of $\alpha$. For some properties, such as the property $P_{H}$ of admitting a copy of $H$, the value of $\alpha$ obtained in the proof of Theorem 1.1, is best possible as well. That is, one can determine the largest value of $q$ for which the $(1: q)$ game $\left(E, \mathcal{F}_{P_{H}}\right)$ is a Maker's win, up to a multiplicative constant factor (we discuss this fact in more detail in Section 4). For general properties, however, this is not the case. Indeed, for the special case in which $P$ is the property of being not-r-colourable, we prove the following stronger result.

Theorem 1.2. Let $r$ be a positive integer and let $P(r)$ be the property of being not-r-colourable (that is, of having chromatic number at least $r+1)$. Let $\varepsilon>0$ and let $G=(V, E)$ be a graph with $n$ vertices and $m$ edges, where $n$ is sufficiently large. If $G$ is $\varepsilon$-robust with respect to $P(r)$, then Maker has a winning strategy for the $(1: q)$ game $\left(E, \mathcal{F}_{P(r)}\right)$, for every $q \leqslant \frac{c \varepsilon^{2} m}{n \log r}$, where $c>0$ is an appropriate constant.

The bound on $q$ given in Theorem 1.2 is not far from being tight, at least for fixed values of $r$. This is discussed further in the final section. 
A different notion of 'robustness' was considered by Duffus, Łuczak and Rödl in [6]. They study the $(1: q)$ Maker-Breaker $\left(E, \mathcal{F}_{P(r)}\right)$ game, where $P(r)$ is the property of being not- $r$ colourable, and $G=(V, E)$ is a graph on $n$ vertices with chromatic number $\chi$. Duffus, tuczak and Rödl asked how large should $\chi$ be in order to ensure that this game is a Maker's win. They conjectured that this value of $\chi$ is independent of $n$. We prove the following weaker result.

Theorem 1.3. Let $q$ and $r$ be positive integers. There exists a constant $c=c(q, r)$ such that, if $G$ is a graph on $n$ vertices and $\chi(G) \geqslant c \log n$, then Maker has a winning strategy for the $(1: q)$ game $\left(E, \mathcal{F}_{P(r)}\right)$.

In fact Duffus, Łuczak and Rödl consider the somewhat different setting, in which $G$ is a hypergraph, and the players claim vertices of $G$. However, they mention that the case where $G$ is a graph and $q=r=2$ is open. In the special case where $G=(V, E)$ is a graph, they consider the game $\left(V, \mathcal{F}_{P(r)}\right)$ rather than $\left(E, \mathcal{F}_{P(r)}\right)$ (that is, the players claim vertices and not edges). However, we feel that, for graphs, the edge version is the more natural one. Still, the result stated in Theorem 1.3 holds for the vertex version as well.

Note that for $q=1$ one can easily obtain a stronger result than that ensured by Theorem 1.3, by using a strategy stealing argument. Indeed, let $G$ be a graph on $n$ vertices satisfying $\chi(G)>r^{2}$. Let $G_{M}$ and $G_{B}=G \backslash G_{M}$ denote the subgraphs of $G$, built by Maker and Breaker, respectively, during the game (played according to certain strategies). Clearly $\chi\left(G_{M}\right) \chi\left(G \backslash G_{M}\right) \geqslant \chi(G)$. Hence, either $\chi\left(G_{M}\right)>r$ or $\chi\left(G_{B}\right)>r$. Assume for the sake of contradiction that no strategy of Maker guarantees $\chi\left(G_{M}\right)>r$. It follows from the above that there exists a strategy $S_{B}$ of Breaker that ensures $\chi\left(G_{B}\right)>r$, regardless of Maker's strategy. However, Maker can 'steal' $S_{B}$, that is, he can claim an arbitrary first edge and then play according to $S_{B}$, pretending to be the second player (whenever he is supposed to claim an edge that is already his, he claims an arbitrary free edge). It follows by the definition of $S_{B}$ that $\chi\left(G_{M}\right)>r$, contrary to our assumption. Note that strategy stealing is a purely existential argument: we do not know of any explicit strategy for Maker that ensures his win in the game with these parameters. On the other hand, Theorem 1.3 gives the currently best bound for any $q \geqslant 2$ and $r \geqslant 2$.

One can apply the Duffus, Łuczak and Rödl notion of robustness to other graph properties besides the property of being not- $r$-colourable. Consider the property $P_{k}$ of being $k$-edgeconnected. Is there a function $f: \mathbb{N} \times \mathbb{N} \rightarrow \mathbb{N}$, such that, for every $f(k, q)$-edge-connected graph $G=(V, E)$, Maker has a winning strategy for the $(1: q)$ game $\left(E, \mathcal{F}_{P_{k}}\right)$ ? For $q=1$ the answer is yes. Indeed, a classical theorem of Nash-Williams [12] and of Tutte [13] asserts that $4 k$-edgeconnectivity ensures the existence of $2 k$ pairwise edge-disjoint spanning trees. These trees, in turn, ensure Maker's win by the classical theorem of Lehman [11]. Hence, $f(k, 1)=4 k$ suffices for every $k \in \mathbb{N}$. For $q \geqslant 2$ and relatively small values of $k$, the answer is no. Indeed, consider a complete bipartite graph $G=(A \cup B, E)$, where $|A|=c \log n$ and $|B|=n-c \log n$, for an appropriate constant $c>0$. By using a Box Game strategy (see [5]), we conclude that Breaker can isolate some vertex of $B$ and thus win the game. For 'large' values of $k$ we prove the following.

Theorem 1.4. Let $G=(V, E)$ be a graph on $n$ vertices, and let $q \geqslant 2$ and $k=k(n) \geqslant \log _{2} n$ be integers. If $G$ is $\left(100 \mathrm{kq} \log _{2} q\right)$-edge-connected, then Maker has a winning strategy for the (1:q) game $\left(E, \mathcal{F}_{P_{k}}\right)$. 
There is a striking relation between the theory of positional games and the theory of random graphs, known as the Erdôs paradigm. Roughly speaking, it asserts that, in some games, playing randomly and playing according to an optimal strategy yield the same outcome. Theorem 1.4 is an example of this paradigm. It asserts, in particular, that if $G=(V, E)$ is $\left(c \log _{2}|V|\right)$-edgeconnected for an appropriate constant $c>0$, then if Maker follows his optimal strategy, he will win the $(1: 2)$ game $\left(E, \mathcal{F}_{P_{1}}\right)$ (that is, succeed in constructing a connected graph), regardless of Breaker's strategy. If, on the other hand, both players play randomly, then the graph built by Maker can be viewed as a random subgraph of $G$ with $|E| / 3$ edges. The fact that such a graph is almost surely connected follows from a result of [1]. The aforementioned assumption on edge connectivity of $G$ is easily seen to be tight for the random game, up to the value of $c$.

\subsection{Notation and preliminaries}

For the sake of simplicity and clarity of presentation, we do not make a particular effort to optimize the constants obtained in our proofs. We also omit floor and ceiling signs whenever these are not crucial. Most of our results are asymptotic in nature, and whenever necessary we assume that $n$ is sufficiently large. Throughout the paper, log stands for the natural logarithm, unless stated otherwise. Our graph-theoretic notation is standard and follows that of [14]. In particular, we use the following.

For a graph $G$, let $V(G)$ and $E(G)$ denote its sets of vertices and edges respectively, and put $v(G)=|V(G)|$ and $e(G)=|E(G)|$. For a set $A \subseteq V(G)$, let $E_{G}(A)$ denote the set of edges of $G$ with both endpoints in $A$, and let $e_{G}(A)=\left|E_{G}(A)\right|$. For disjoint sets $A, B \subseteq V(G)$, let $E_{G}(A, B)$ denote the set of edges of $G$ with one endpoint in $A$ and one endpoint in $B$, and let $e_{G}(A, B)=$ $\left|E_{G}(A, B)\right|$. Sometimes, if there is no risk of confusion, we discard the subscript $G$ in the above notation. For a set $S \subset V(G)$, let $\bar{S}=V(G) \backslash S$. Let $(S, \bar{S})$ denote the edge-cut that separates $S$ from $\bar{S}$, that is, $(S, \bar{S})=E_{G}(S, \bar{S})$. For a set $S \subseteq V(G)$, let $G[S]$ denote the subgraph of $G$ induced on the vertices of $S$. For a set $E^{\prime} \subseteq E(G)$, let $G\left[E^{\prime}\right]$ denote the subgraph of $G$ spanned by the edges of $E^{\prime}$. For a graph $H$, let

$$
m_{2}(H):=\max \left\{\frac{e(G)-1}{v(G)-2}: G \subseteq H, v(G) \geqslant 3\right\}
$$

denote its 2-density. Let $G=(V, E)$ be a graph with $m$ edges, and let $0 \leqslant t \leqslant m$ be an integer. The random graph $G(t)$ is the graph obtained from $G$ by randomly deleting $m-t$ edges from $G$ uniformly among all elements of $\left(\begin{array}{c}E \\ m-t\end{array}\right)$. For positive integers $r$ and $k$, and for a fixed graph $H$, let $P(r), P_{k}$ and $P_{H}$ denote the property of being not- $r$-colourable, the property of being $k$-edge-connected, and the property of admitting a copy of $H$, respectively.

The following fundamental theorem, due to Beck [3], is a useful sufficient condition for Breaker's win in the $(p: q)$ game $(X, \mathcal{F})$.

Theorem 1.5. Let $X$ be a finite set and let $\mathcal{F} \subseteq 2^{X}$. If $\sum_{B \in \mathcal{F}}(1+q)^{-|B| / p}<\frac{1}{1+q}$, then Breaker (as first or second player) has a winning strategy for the $(p: q)$ game $(X, \mathcal{F})$.

The rest of the paper is organized as follows. In Section 2 we prove Theorems 1.1 and 1.2, and in Section 3 we prove Theorems 1.3 and 1.4. Finally, in Section 4 we present some open problems. 


\section{Monotone properties}

Proof of Theorem 1.1. Maker's strategy is to build a graph that contains a relatively small subgraph satisfying $P$. Since $P$ is monotone increasing, this will ensure his win. The existence of such a subgraph is guaranteed by the main result of [2].

Theorem 2.1. Let $\varepsilon>0$ and let $P$ be a monotone increasing property. Let $G$ be a graph with $n$ vertices and $\Theta\left(n^{2}\right)$ edges, which is $\varepsilon$-robust with respect to $P$. Then there exists a graph $H$ on $h=h(\varepsilon, P)$ vertices that satisfies $P$, and a constant $\gamma=\gamma(\varepsilon, P)>0$, such that $G$ contains at least $\gamma n^{h}$ copies of $H$.

Remark. In [2], this result is stated in terms of monotone decreasing properties and $\varepsilon$-farness. The statement of Theorem 2.1, as it appears here, is obtained via the equivalence indicated in the previous remark (see page 2).

If there exists a forest $F$ that satisfies $P$, then Maker wins regardless of his strategy, for every sufficiently large $n$ and $q=o(n)$. Indeed, Maker's graph will have $\omega(n)$ edges, and will thus contain every fixed forest. Hence, we assume that $P$ contains no forests. Let $H$ and $\gamma$ be as in Theorem 2.1; by the above we can assume that $H$ contains a cycle. We will prove that Maker can claim a copy of $H$ in $G$. In order to do so, we need the following lemma.

Lemma 2.2. Let $G=(V, E)$ be a graph with $n$ vertices and $m \geqslant c_{1} n^{2}$ edges, where $c_{1}>0$ is a constant. Let $H$ be a fixed graph on $h$ vertices that contains a cycle, and let $0<\gamma<1$ be a constant. Assume that $G$ contains at least $\gamma n^{h}$ copies of $H$. Then, there exist constants $c_{2}>0$ and $0<\delta<1$ such that, with probability at least $2 / 3$, a random graph $G(t)$ with $t=c_{2} m n^{-1 / m_{2}(H)}$ edges is such that every subgraph of it with at least $(1-\delta)$ t edges contains a copy of $H$.

The proof of Lemma 2.2 is a straightforward adaptation of the proof of a lemma of Bednarska and Łuczak (see Lemma 4 in [4]), where a similar result is proved for the special case $G=K_{n}$. For the sake of completeness we include a short sketch of the proof.

Proof. We will use the following fact, which can be proved through standard methods: for $t^{\prime}:=t / 2$, there exists a constant $c^{\prime}>0$ such that $\operatorname{Pr}\left(H \nsubseteq G\left(t^{\prime}\right)\right) \leqslant e^{-c^{\prime} t}$.

Let $0<\delta<1 / 2$ be a constant, small enough to satisfy $\delta-\delta \log \delta<c^{\prime}$. We count pairs $\left(F, F^{\prime}\right)$ such that $F$ is a subgraph of $G$ with $t$ edges, and $F^{\prime}$ is a subgraph of $F$ with $(1-\delta) t \geqslant t^{\prime}$ edges that does not contain a copy of $H$. Counting from the viewpoint of $F^{\prime}$ and using the above fact, we conclude that the number of such pairs is at most

$$
e^{-c^{\prime} t}\left(\begin{array}{c}
m \\
(1-\delta) t
\end{array}\right)\left(\begin{array}{c}
m-(1-\delta) t \\
\delta t
\end{array}\right) .
$$

Hence, we conclude that the number of such pairs is at most

$$
\begin{aligned}
\left(\begin{array}{c}
m \\
t
\end{array}\right)\left(\begin{array}{c}
t \\
\delta t
\end{array}\right) e^{-c^{\prime} t} & \leqslant(e / \delta)^{\delta t} e^{-c^{\prime} t}\left(\begin{array}{c}
m \\
t
\end{array}\right) \\
& \leqslant \frac{1}{3}\left(\begin{array}{c}
m \\
t
\end{array}\right),
\end{aligned}
$$


where the last inequality holds for sufficiently large $n$. It follows that the probability that $G(t)$ contains a subgraph on $(1-\delta) t$ vertices which does not contain a copy of $H$ is at most $1 / 3$, as claimed.

We are now ready to describe Maker's strategy. Let $c_{2}>0$ and $0<\delta<1$ be the constants whose existence is guaranteed by Lemma 2.2, and let $q=\frac{\delta}{2 c_{2}} n^{1 / m_{2}(H)}-1$. In the first $t:=$ $\delta m /(2 q+2)=c_{2} m n^{-1 / m_{2}(H)}$ rounds, Maker plays randomly, that is, before the game starts Maker draws uniformly at random $t$ edges of $G$. Let $\left\{e_{i}\right\}_{i=1}^{t}$ be an arbitrary ordering of the chosen edges; these edges are kept secret from Breaker until they are claimed. In his $i$ th move, for $1 \leqslant i \leqslant t$, Maker claims the edge $e_{i}$. If $e_{i}$ has been previously claimed by him or by Breaker, then he claims an arbitrary free edge. If $e_{i}$ is claimed by Breaker, then it is declared a failure. In his $i$ th move, for $i>t$, Maker claims an arbitrary free edge.

Let $S_{M}$ denote the aforementioned strategy of Maker, and let $S_{B}$ be an arbitrary fixed strategy of Breaker. We claim that if Maker follows $S_{M}$ and Breaker follows $S_{B}$, then Maker wins with positive probability. First we prove the following lemma.

\section{Lemma 2.3. With probability at least $1 / 2$, there are at most $\delta t$ failures.}

Proof. During the first $t$ rounds, both players claim together at most $t(q+1)=\delta m / 2$ edges. It follows that, for every $1 \leqslant i \leqslant t$, the probability that $e_{i}$ is a failure is at most $\delta / 2$. Let $X$ be the random variable that counts the number of failures. Then $\mathbb{E}(X) \leqslant \delta t / 2$. It follows by Markov's inequality that $\operatorname{Pr}(X \geqslant \delta t) \leqslant 1 / 2$.

We are now ready to prove our claim. Let $M_{t}$ denote the graph built by Maker during the first $t$ rounds and let $\tilde{M}_{t}:=M_{t} \cap\left\{e_{1}, \ldots, e_{t}\right\}$. The graph $\tilde{M}_{t}$ can be viewed as a random graph $G(t)$ from which an adversary has removed some edges, namely, all the edges that were declared a failure. By Lemma 2.3, with probability at least $1 / 2$ there are at most $\delta t$ failures, that is, there are at least $(1-\delta) t$ edges in $\tilde{M}_{t}$. Assuming $e\left(\tilde{M}_{t}\right) \geqslant(1-\delta) t$, it follows by Lemma 2.2 that $\tilde{M}_{t}$ contains a copy of $H$ with probability at least $2 / 3$. Thus, $\tilde{M}_{t}$ contains a copy of $H$ with probability at least $1 / 6$.

Hence, with positive probability, Maker has won the game. Since this is a finite perfect information game with no chance moves, it follows that there exists a deterministic winning strategy for Maker to win this game, assuming Breaker follows $S_{B}$. However, since $S_{B}$ was arbitrary, this holds for any strategy of Breaker. It follows that the game is a Maker's win, as claimed.

Proof of Theorem 1.2. Maker plays as follows. Let $\delta=\varepsilon / 4$. In the first $t:=\delta m /(q+1)$ rounds, Maker plays randomly, that is, before the game starts Maker draws uniformly at random $t$ edges of $G$. Let $\left\{e_{i}\right\}_{i=1}^{t}$ be an arbitrary ordering of the chosen edges; these edges are kept secret from Breaker until they are claimed. In his $i$ th move, for $1 \leqslant i \leqslant t$, Maker claims the edge $e_{i}$. If $e_{i}$ has been previously claimed by him or by Breaker, then he claims an arbitrary free edge. If $e_{i}$ is claimed by Breaker, then it is declared a failure. In his $i$ th move, for $i>t$, Maker claims an arbitrary free edge. 
Denote the aforementioned strategy of Maker by $S_{M}$, and let $S_{B}$ be an arbitrary fixed strategy of Breaker. We claim that if Maker follows $S_{M}$ and Breaker follows $S_{B}$, then Maker wins with positive probability. First we prove two lemmas.

Lemma 2.4. Let $G=(V, E)$ be as in Theorem 1.2. Let $\gamma=\delta /(q+1)$, and let $R \subseteq E$ be a set of size $\gamma m$, drawn uniformly at random among all such sets. Then, with probability at least $2 / 3$, $G_{R}:=(V, R)$ is $\varepsilon / 2$-robust with respect to being not-r-colourable, that is, one has to delete at least $\varepsilon \gamma \mathrm{m} / 2$ edges from $G_{R}$ in order to obtain an r-colourable graph.

Proof. Let $V=V_{1} \cup \cdots \cup V_{r}$ be an arbitrary fixed partition of $V$ into $r$ parts. Since $G$ is $\varepsilon^{-}$ robust with respect to being not-r-colourable, it follows that $f:=\sum_{i=1}^{r} e_{G}\left(V_{i}\right) \geqslant \varepsilon m$. Let $Y=$ $\sum_{i=1}^{r} e_{G_{R}}\left(V_{i}\right)$. Then $Y$ is a hypergeometric random variable with parameters $m, f$ and $\gamma m$; in particular, $\mathbb{E}(Y)=\gamma f \geqslant \varepsilon \gamma m$. It follows by standard bounds on the tail of the hypergeometric distribution (see, e.g., [8]) that

$$
\begin{aligned}
\operatorname{Pr}(Y \leqslant \varepsilon \gamma m / 2) & \leqslant \operatorname{Pr}(Y \leqslant \mathbb{E}(Y) / 2) \\
& \leqslant e^{-\varepsilon \gamma m / 8} \\
& \leqslant \frac{1}{3} r^{-n},
\end{aligned}
$$

where the last inequality follows by the upper bound on $q$, assumed in Theorem 1.2. Since there are at most $r^{n}$ such partitions, the result follows by a union bound argument.

Lemma 2.5. The probability that there are at least $\varepsilon \delta m /(2 q+2)$ failures is at most $1 / 2$.

Proof. During the first $t$ rounds, both players claim together at most $t(q+1)=\delta m$ edges. It follows that, for every $1 \leqslant i \leqslant t$, the probability that $e_{i}$ is a failure is at most $\delta$. Let $X$ be the random variable that counts the number of failures. Then $\mathbb{E}(X) \leqslant \delta t=\delta^{2} m /(q+1)$. It follows by Markov's inequality that $\operatorname{Pr}(X \geqslant \varepsilon \delta m /(2 q+2)) \leqslant 1 / 2$.

We are now ready to prove our claim. Let $M_{t}$ denote the graph built by Maker during the first $t$ rounds and let $\tilde{M}_{t}:=M_{t} \cap\left\{e_{1}, \ldots, e_{t}\right\}$. The graph $\tilde{M}_{t}$ can be viewed as a random graph $G(t)$ from which an adversary has removed some edges, namely, all the edges that were declared a failure. It follows by Lemma 2.4 that the probability that one has to delete at least $\varepsilon \delta m /(2 q+2)$ edges from $G(t)$ in order to obtain an $r$-colourable graph is at least $2 / 3$. However, by Lemma 2.5 , with probability at least $1 / 2$, a smaller number of edges were removed from $G(t)$ to obtain $\tilde{M}_{t}$. Thus, $\tilde{M}_{t}$ is not $r$-colourable with probability at least $1 / 6$.

Hence, with positive probability, Maker has won the game. Since this is a finite perfect information game with no chance moves, it follows that there exists a deterministic winning strategy for Maker to win this game, assuming Breaker follows $S_{B}$. However, since $S_{B}$ was arbitrary, this holds for any strategy of Breaker. It follows that the game is a Maker's win, as claimed.

\section{Chromatic number and edge connectivity}

Proof of Theorem 1.3. We first prove a few simple but useful facts. 
Proposition 3.1. Let $q \geqslant 1$ and $r \geqslant 2$ be integers and let $G=(V, E)$ be a graph on $n$ vertices, where $n$ is sufficiently large. Each of the following is a sufficient condition for Maker's win in the $(1: q)$ game $\left(E, \mathcal{F}_{P(r)}\right)$.

(a) Maker can win the same game on some subgraph of $G$.

(b) G contains a clique of size $1000 q r \log r$.

(c) $\alpha(G) \leqslant n /(10 q r \log r)$.

Proof. (a) Let $H$ be a subgraph of $G$ on which Maker can win the $(1: q)$ game $\left(E(H), \mathcal{F}_{P(r)}\right)$. Let $S_{H}$ be an arbitrary winning strategy of Maker in this game. When playing the $(1: q)$ game $\left(E, \mathcal{F}_{P(r)}\right)$, Maker follows $S_{H}$ while ignoring $E \backslash E(H)$. That is, if in some move Breaker claims $q^{\prime}<q$ edges of $E(H)$, then Maker (in his mind) gives Breaker $q-q^{\prime}$ additional free edges of $E(H)$, and then plays according to $S_{H}$. Clearly, by the time every edge of $E(H)$ is claimed, Maker has built a non-r-colourable subgraph of $H$, which is also a non- $r$-colourable subgraph of $G$.

(b) Let $s=1000 q r \log r$ and let $H$ be a copy of $K_{s}$ in $G$. It was proved in [7] that if $q \leqslant \frac{\ell}{1000 r \log r}$, then, by playing a $(1: q)$ game on the edge set of $K_{\ell}$, Maker can build a graph with chromatic number at least $r+1$. The claim now follows by condition (a) of this proposition.

(c) Let $s=10 q r \log r$, and assume that $\alpha(G) \leqslant n / s$. Maker's goal is to build a graph that does not admit an independent set of size $n / r$, and so, in particular, is not $r$-colourable. Let $A \subseteq V$ be an arbitrary set of size $n / r$. By our assumption $\alpha(G[A]) \leqslant n / s$. It follows by Turán's theorem that $e(G[A]) \geqslant 4.5 q n \log r / r$. Let $\mathcal{I}_{G}$ denote the family of edge-sets of all induced subgraphs of $G$ on $n / r$ vertices. It is clear that if Breaker can win the $(q: 1)$ game $\left(E, \mathcal{I}_{G}\right)$, then Maker can win the $(1: q)$ game $\left(E, \mathcal{F}_{P(r)}\right)$ by ensuring that the independence number of his graph is strictly smaller than $n / r$. In order to prove that Breaker can win $\left(E, \mathcal{I}_{G}\right)$, we apply Theorem 1.5 . We have

$$
\begin{aligned}
\sum_{B \in \mathcal{I}_{G}} 2^{-|B| / q} & \leqslant\left(\begin{array}{c}
n \\
n / r
\end{array}\right) 2^{-4.5 n \log r / r} \\
& \leqslant(e r)^{n / r} e^{-3 n \log r / r} \\
& \leqslant\left(e^{1-2 \log r}\right)^{n / r} \\
& =o(1),
\end{aligned}
$$

where the last equality holds since $r \geqslant 2$.

We are now ready to prove Theorem 1.3. We assume $r \geqslant 2$ as the assertion of the theorem is trivial for $r=1$.

Let $a=10 q r \log r$ and let $k=k(q, r)$ be the smallest positive integer such that, for every graph $G$ on $n \geqslant k$ vertices satisfying $\alpha(G) \leqslant n / a$, Maker has a winning strategy for the $(1: q)$ game $\left(E(G), \mathcal{F}_{P(r)}\right)$ (the existence of such an integer $k$ is guaranteed by part (c) of Proposition 3.1). Let $b=\frac{a}{a-1}$, and let $n_{0}$ be the largest integer satisfying $n_{0} \leqslant k+\log _{b} n_{0}$. For every $i \geqslant 0$, let $n_{i}=n_{0} b^{i}$.

Claim 3.2. Let $i \geqslant 0$ be an integer, and let $G$ be a graph on $n_{i}$ vertices such that $\chi(G) \geqslant k+$ $\log _{b} n_{i}$. Then there exists a subgraph $G^{*} \subseteq G$ such that $v\left(G^{*}\right) \geqslant k$ and $\alpha\left(G^{*}\right) \leqslant v\left(G^{*}\right) / a$. 
Proof. We proceed by induction on $i$. The claim clearly holds for $i=0$, as then we have $v(G)=$ $n_{0} \leqslant k+\log _{b} n_{0} \leqslant \chi(G)$. It follows that $G$ is a complete graph and thus $\alpha(G)=1$. Hence, since $n_{0} \geqslant k \geqslant a$, we conclude that $G^{*}:=G$ satisfies the assertion of the claim. Next, let $G$ be a graph on $n_{i}$ vertices, for some $i \geqslant 1$, such that $\chi(G) \geqslant k+\log _{b} n_{i}$. If $\alpha(G) \leqslant n_{i} / a$, then $G^{*}:=G$ satisfies the assertion of the claim. Otherwise, let $I \subseteq V(G)$ be an arbitrary independent set of size $n_{i} / a$, and let $G^{\prime}=G[V(G) \backslash I]$. Note that $v\left(G^{\prime}\right)=n_{i}-n_{i} / a=n_{i} b^{-1}=n_{i-1}$ and $\chi\left(G^{\prime}\right) \geqslant$ $\chi(G)-1 \geqslant k+\log _{b} n_{i}-\log _{b} b=k+\log _{b} n_{i-1}$. Hence, by the induction hypothesis, there exists a subgraph $G^{*} \subseteq G^{\prime} \subseteq G$ such that $v\left(G^{*}\right) \geqslant k$ and $\alpha\left(G^{*}\right) \leqslant v\left(G^{*}\right) / a$.

Let $G$ be a graph on $n>n_{0}$ vertices, such that $\chi(G) \geqslant k+\log _{b} n+1$. Let $i$ be the integer for which $n_{i}<n \leqslant n_{i+1}$. Let $G^{\prime}$ be the graph obtained from $G$ by adding $n_{i+1}-n$ isolated vertices. Note that $v\left(G^{\prime}\right)=n_{i+1}$ and $\chi\left(G^{\prime}\right)=\chi(G) \geqslant k+\log _{b} n_{i+1}$. It follows by Claim 3.2 that there exists a subgraph $G^{*} \subseteq G^{\prime}$ such that $v\left(G^{*}\right) \geqslant k$ and $\alpha\left(G^{*}\right) \leqslant v\left(G^{*}\right) / a$. Hence, by the choice of $k$, it follows that Maker has a winning strategy for the $(1: q)$ game $\left(E\left(G^{*}\right), \mathcal{F}_{P(r)}\right)$. By part (a) of Proposition 3.1 we conclude that Maker also wins this game when played on $G^{\prime}$. Since $G^{\prime} \backslash G$ consists of isolated vertices, it follows that the $(1: q)$ game $\left(E(G), \mathcal{F}_{P(r)}\right)$ is a Maker's win, as claimed.

Proof of Theorem 1.4. We will make use of the following theorem of Karger [10].

Theorem 3.3. Let $G=(V, E)$ be a graph on $n$ vertices, which is r-edge-connected. Then, for every $t \geqslant 1$, the number of cuts of size at most $r t$ in $G$ is at most $\mathrm{cn}^{2 t}$ for some positive constant $c$.

Consider the following auxiliary Maker-Breaker game $(E, \mathcal{F})$, which we refer to as the $C u t$ Game. Two players, called CutMaker and CutBreaker, take turns in claiming free edges of $G=$ $(V, E)$. CutBreaker, who is the first player, claims 1 edge per turn, whereas CutMaker claims $q$ edges. The family $\mathcal{F}$ consists of all edge-sets $L \subseteq E$ for which there exists a cut $(S, \bar{S})$ of $G$ such that $L \subseteq E(S, \bar{S})$ and $|L|=e(S, \bar{S})-k+1$. CutMaker wins the game if he claims all edges of some element of $\mathcal{F}$; otherwise CutBreaker wins. It is easy to see that the $(1: q)$ game $\left(E, \mathcal{F}_{P_{k}}\right)$ is a Maker's win if and only if the $(q: 1)$ Cut Game is a CutBreaker's win. By Theorem 3.3 we have

$$
\begin{aligned}
\sum_{B \in \mathcal{F}} 2^{-|B| / q} & \leqslant \sum_{i=100 k q \log _{2} q}^{n^{2}}|\{S \subset V: e(S, \bar{S})=i\}|\left(\begin{array}{c}
i \\
k-1
\end{array}\right) 2^{-(i-k+1) / q} \\
& \leqslant \sum_{i=100 k q \log _{2} q}^{n^{2}} c n^{\frac{2 i}{100 k q \log _{2} q}}(e i / k)^{k} 2^{(k-i) / q} \\
& \leqslant c \sum_{i=100 k q \log _{2} q}^{n^{2}} 2^{\frac{i}{50 q}-\frac{i}{2 q}+2 k \log (i / k)} \\
& =o(1),
\end{aligned}
$$

where the third inequality holds since $k \geqslant \log _{2} n$, and the last equality holds since $k=\omega(1)$. 
Hence, it follows by Theorem 1.5 that the $(q: 1)$ game $(E, \mathcal{F})$ is indeed a CutBreaker's win.

\section{Concluding remarks and open problems}

(1) In Theorem 1.1, we have proved that there exists a winning strategy for Maker in the $(1: q)$ game $\left(E, \mathcal{F}_{P}\right)$ for every dense graph $G=(V, E)$ on $n$ vertices that is robust with respect to $P$, and for every $q \leqslant c n^{\alpha}$ for some constants $c>0$ and $\alpha>0$. For the special case in which $P=P(r)$ is the property of being not- $r$-colourable, Theorem 1.2 provides a stronger result: namely, the requirement that $G$ is dense becomes redundant, and the upper bound on $q$ is improved to $c(r) \frac{|E|}{|V|}$. Both theorems are existential in nature. It would be interesting to find explicit, efficient and deterministic winning strategies for Maker in these games, under similar conditions.

(2) As mentioned in the Introduction, Theorem 1.1 is essentially best possible for some properties. One witness of this fact is the property $P_{H}$ of admitting a copy of $H$, for some fixed graph $H$. Indeed, let $G=(V, E)$ be a graph with $n$ vertices and $\Theta\left(n^{2}\right)$ edges, which is $\varepsilon$-robust with respect to $P_{H}$. It is well known that $G$ contains $\gamma n^{v(H)}$ copies of $H$, where $\gamma>0$ is a constant. Hence, it follows from the proof of Theorem 1.1 that Maker has a winning strategy for the $(1: q)$ game $\left(E, \mathcal{F}_{P_{H}}\right)$ for every $q \leqslant c_{1} n^{1 / m_{2}(H)}$, where $c_{1}>0$ is an appropriate constant. Now assume that $q \geqslant c_{2} n^{1 / m_{2}(H)}$ for some sufficiently large constant $c_{2}$. We claim that for such a $q$, Breaker has a winning strategy for the $(1: q)$ game $\left(E, \mathcal{F}_{P_{H}}\right)$. Clearly it suffices to prove this for the $(1: q)$ game $\left(E\left(K_{n}\right), \mathcal{F}_{P_{H}}\right)$. This, however, has been proved in [4]. On the other hand, Theorem 1.2 shows that for some properties, such as being not-r-colourable, the upper bound given in Theorem 1.1 is far from being best possible. It would be interesting to obtain tighter bounds for additional monotone properties.

(3) The bound on $q$ given in Theorem 1.2 is not far from being tight, at least for fixed values of $r$. Indeed, consider for example the graph $G$, which consists of $n / d$ disjoint copies of $K_{d}$, where $n \gg d>r=3$. Clearly, one has to delete a constant fraction of the edges of every $d$-clique of $G$ in order to obtain an $r$-colourable graph. Thus $G$ is $\Omega(1)$-robust with respect to being not- $r$ colourable. However, if $q \geqslant 2 d /(r-2)$, then Breaker can make sure that the maximum degree in Maker's graph will be at most $r-1$, and thus Maker's graph will be $r$-colourable. Breaker's strategy is very simple: whenever Maker claims an edge $(u, v)$, Breaker responds by claiming $q / 2$ arbitrary free edges that are incident with $u$ and $q / 2$ arbitrary free edges that are incident with $v$. For large $r$, the maximum possible value of $\varepsilon$ is only $\Theta(1 / r)$, as any graph can be made $r$-colourable by deleting at most an $O(1 / r)$ fraction of its edges. Here, however, one can show that for $q \geqslant C d /(r \log r)$, Breaker has a winning strategy for some graphs. Indeed, let $d$ be an integer satisfying $d \gg r \log r$. Let $G=G(n, d / n)$ be a random graph on $n$ vertices. Delete from $G$ all edges that are incident with vertices of degree higher than $2 d$; denote the resulting graph by $G^{\prime}=(V, E)$. One can easily show that $|V|=n$, and almost surely $|E|=\Theta(d) n$ and $G^{\prime}$ is $\Theta(1 / r)$ robust with respect to being not- $r$-colourable. However, if $q \geqslant C d /(r \log r)$, for an appropriate constant $C>0$, then Breaker can make sure that Maker's graph will be triangle-free, and with maximum degree at most $\tilde{c} r \log r$, where $\tilde{c}>0$ is sufficiently small. It follows by Johansson's theorem [9] that Maker's graph will be $r$-colourable. Breaker plays as follows: in each move he first claims every free edge that closes a triangle in Maker's graph. Denote the number of 
such edges by $q_{1}$ and let $q_{2}=q-q_{1}$. Since the triangles in $G(n, d / n)$ are almost surely edgedisjoint, we can assume in fact that $q_{1}=1$. Then, if the last edge claimed by Maker was $(u, v)$, then Breaker responds by claiming $q_{2} / 2$ arbitrary free edges that are incident with $u$ and $q_{2} / 2$ arbitrary free edges that are incident with $v$. This strategy is quite similar to that suggested by Chvátal and Erdős in [5] (see Theorem 5.2 there).

\section{Acknowledgement}

We would like to thank the anonymous referee for helpful comments.

\section{References}

[1] Alon, N. (1995) A note on network reliability. In Discrete Probability and Algorithms (D. Aldous, P. Diaconis, J. Spencer and J. M. Steele, eds), Vol. 72 of IMA Volumes in Mathematics and its Applications, Springer, pp. 11-14.

[2] Alon, N. and Shapira, A. (2005) Every monotone graph property is testable. In Proc. 37 ACM STOC, Baltimore, ACM Press, pp. 128-137. Also: SICOMP (Special Issue of STOC'05) 38 (2008) 505-522.

[3] Beck, J. (1982) Remarks on positional games. Acta Math. Acad. Sci. Hungar. 40 65-71.

[4] Bednarska, M. and Łuczak, T. (2000) Biased positional games for which random strategies are nearly optimal. Combinatorica 20 477-488.

[5] Chvátal, V. and Erdős, P. (1978) Biased positional games. Ann. Discrete Math. 2 221-228.

[6] Duffus, D., Łuczak, T. and Rödl, V. (1998) Biased positional games on hypergraphs. Studia Sci. Math. Hungar. 34 141-149.

[7] Hefetz, D., Krivelevich, M., Stojaković, M. and Szabó, T. (2008) Planarity, colorability and minor games. SIAM J. Discrete Math. 22 194-212.

[8] Janson, S., Łuczak, T. and Ruciński, A. (2000) Random Graphs, Wiley, New York.

[9] Johansson, A. (1996) Asymptotic choice number for triangle free graphs. DIMACS Technical Report 91-95.

[10] Karger, D. (1993) Global min-cuts and other ramifications of a simple min-cut algorithm. In Proc. 4th Annual ACM-SIAM Symposium on Discrete Algorithms (Austin TX), ACM, New York, pp. 21-30.

[11] Lehman, A. (1964) A solution of the Shannon switching game. J. Soc. Indust. Appl. Math. 12 687-725.

[12] Nash-Williams, C. S. J. A. (1961) Edge disjoint spanning trees of finite graphs. J. London Math. Soc. 36 445-450.

[13] Tutte, W. T. (1961) On the problem of decomposing a graph into $n$ connected factors. J. London Math. Soc. 36 221-230.

[14] West, D. B. (2001) Introduction to Graph Theory, Prentice-Hall. 